\title{
Analytical Landing Trajectories For Embedded Autonomy
}

\author{
Maodeng $\mathrm{Li}^{1,2, *}$, Malcolm Macdonald ${ }^{1}$, C. R. McInnes ${ }^{1}$ and Wuxing Jing ${ }^{2}$ \\ ${ }^{1}$ Advance Space Concepts Laboratory, University of Strathclyde, Glasgow, Scot- \\ land, E.U. \\ ${ }^{2}$ Department of Aerospace Engineering, Harbin Institute of Technology, Harbin, \\ Heilongjiang, China.
}

\section{ABSTRACT}

This paper considers an optimal guidance law for the initial braking phase of a soft landing mission on a celestial body without atmosphere in which boundary conditions on height and velocity are specified. The optimal lander attitude for the minimum fuel landing problem is found. An analytic optimal trajectory is achieved by expanding the thrust acceleration, gravitational acceleration and the cosine of the vertical attitude angle to a high-order polynomial. Coefficients of these polynomials are obtained from the boundary conditions. A fixed gain control law and a direct adaptive control law are then developed to track the analytical reference trajectory. Finally, a mission scenario is presented to illustrate the accuracy of the analytical trajectory and validity of the control laws developed. The use of direct adaptive control for embedded autonomy will be directly contrasted against a traditional fixed gain controller, using a Lunar landing scenario. The advantage of the direct adaptive control approach is that it does not require system monitoring to detect thruster failure and can adjust its gain automatically. As such, direct adaptive control combined with the developed analytical solution enables autonomy to be embedded within the lander guidance and control system. In addition, it is shown that direct adaptive control increases the probability of lander survival through faster transient response and stability than a traditional fixed gain controller with system level

\footnotetext{
* Corresponding author: Advance Space Concepts Laboratory, University of Strathclyde, James Weir Building, 75 Montrose Street, Glasgow, G1 1XJ, Scotland, E.U.

email: mdeng1985@gmail.com
} 
failure detection and recovery.

\section{INTRODUCTION}

Guidance and control plays a key role in soft landing missions. To design the guidance and control law, two factors should be taken into consideration to ensure missions success: a low touch down velocity and a vertical attitude on the planetary surface. Many studies on guidance for soft landing have been reported [1-14]. These guidance laws can be divided into two catalogues:

1.) Gravity-Turn guidance law $[1-10,12]$. Such a descent profile requires that the vehicle thrust vector is oriented opposite to the instantaneous velocity vector along the descent trajectory. To develop a gravity-turn guidance law, an approximate form solution is first required [1-4], and a control law is then designed to track this predefined profile [3-9]. The following methods have been developed successfully: linear feedback control $[5,6]$, nonlinear control via nonlinear transformation [7], direct adaptive control (DAC) [8], feedback linearization [9] and methods using Lyapunov stability theory $[3,4]$. Gravity-turn descent has been used in practice for terminal descent to planetary surface [8]. An important benefit of gravity-turn descent is that the landing is assured to be vertical [6]. This guidance law, however, is not derived from optimal control theory, thus fuel consumption is not minimum [10].

2.) Tangent guidance Law [10-14]. This guidance law is derived from optimal control. Based on some assumptions (i.e., mass of lander or gravitational acceleration is assumed to be constant), an analytic optimal solution for 2-D planetary landing problem required for fuzzy training was previously determined using regular perturbation theorem, and then a fuzzy algorithm was followed to achieve the closed-loop guidance strategy [11]. However, in general, a closed form solution for this guidance law cannot be found for the full model [12]. Some methods have been developed to approach the optimal solution. A typical approach of calculating the trajectory is to develop an analytic profile that closely approximates to the optimal solution. This can be accomplished by 
restricting the acceleration profile to a polynomial function in each axis (constant $[10,13,14]$, linear $[10,12,13]$, or quadratic $[12,14])$, the trajectory is then shaped by selecting a set of boundary conditions, which are used to solve for the coefficients of the guidance equations. The main drawback of this approach is that there exists no guarantee under arbitrary conditions for convergence [3]. Within this paper the tangent guidance law is mainly considered, presenting a solution to convergence issues and bridging the gap between this guidance law and the gravity turn guidance law. Using the optimal attitude angles of the minimum fuel landing problem, the thrust acceleration, gravitational acceleration and the cosine of the vertical attitude angle are expanded to high-order polynomials. An analytical solution for 3-D planetary landing problem is approximated using polynomial iteration methods. The coefficients of these polynomials are solved from the boundary conditions. Numerical results show that the analytical solution is a good match to the numerical solution. Compared to numerical results, the analytical solution is an explicit function of time. Furthermore, it should be noted that due to the inherent closed form nature of the derived solution it is suitable for on-board implementation within the guidance system. In addition to this analytical contribution, a robust DAC law is presented which is proved to be convergent using Lyapunov stability theory. The DAC law is shown to be resilient to variations in the available thrust level, continuing to track the derived closed form polynomial solution in the presence of such variations. As such, the combination of on-board implementation of the derived high-accuracy closed form polynomial solution within the guidance system, with the highly robust DAC introduces and enables the concept of embedded autonomy to mission critical control systems. Compared to fixed gain control (FGC), DAC does not require systems monitoring to detect variations in thrust level availability, thus enabling the on-board monitoring of this system to be replaced with embedded autonomy. Furthermore, DAC exhibits fast transient response and stability, thus if thruster failure occurs near the terminal time the lander will crash for FGC, but survive for DAC. As such, DAC increases the probability of lander survival. The paper is organized as follows. Initially, the dimensional 
and dimensionless equations of motion are derived. Subsequently the tangent guidance law which is a linear function of time is introduced, and then analytical optimal solution is derived from the dimensionless equations. Thereafter, the FGC and DAC approach for tracking height and horizontal velocity are derived. Finally, a sample mission scenario is presented to illustrate the ability of the guidance and control system through numerical simulation. The reader should note that a Lunar scenario is used within this paper to illustrate the developed techniques, however they remain suitably generic for application to other bodies.

\section{SYSTEM MODELS}

The idealized assumptions of a point mass lander and a regular spherical planet without atmosphere are made in this paper. Figure 1 illustrates the geometry and variables of the problem. As seen in Figure 1, a planetary fixed rotating frame, denoted by $\mathbb{F}_{P}$, has its origin located in the center of the planetary, the $z_{P}$ axis is directed along the planet's rotation axis towards the celestial north pole, and the $x_{P}$ axis intersects the zero latitude, zero longitude point on the equator. The $y_{P}$ axis completes a right handed orthogonal frame. The vehicle carried local vertical frame, denoted by $\mathbb{F}_{H}$, has its origin located in the center of the mass of the lander. The $z_{H}$ axis points towards the planet's center, $x_{H}$ points North, and $y_{H}$ points East. 


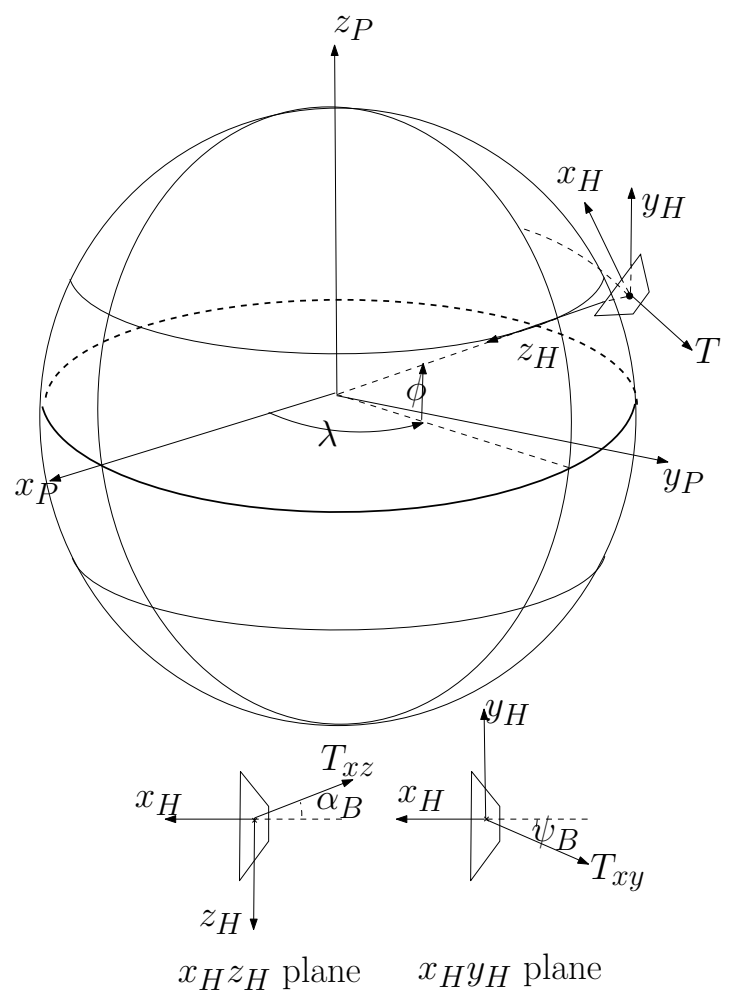

Fig. 1 Geometry and variables of planetary landing problem 


\subsection{Dimensional Model}

The position of the lander $[r, \phi, \lambda]^{\top}$ is described in $\mathbb{F}_{P}$, with the lander's velocity $[u, v, w]^{\top}$ expressed in $\mathbb{F}_{H}$. The equations of motion are given as $[9,10]$

$$
\begin{aligned}
\dot{r} & =-w \\
\dot{\phi} & =\frac{1}{r} u \\
\dot{\lambda} & =\frac{1}{r \cos \phi} v \\
\dot{u} & =-\frac{v^{2}}{r} \tan \phi+\frac{u w}{r}+\frac{T_{x}}{m} \\
\dot{v} & =\frac{u v}{r} \tan \phi+\frac{v w}{r}+\frac{T_{y}}{m} \\
\dot{w} & =-\frac{u^{2}+v^{2}}{r}+\frac{\mu}{r^{2}}+\frac{T_{z}}{m}
\end{aligned}
$$

where,

$$
\left[\begin{array}{c}
T_{x} \\
T_{y} \\
T_{z}
\end{array}\right]=\left[\begin{array}{c}
-T \cos \alpha_{B} \cos \psi_{B} \\
-T \cos \alpha_{B} \sin \psi_{B} \\
-T \sin \alpha_{B}
\end{array}\right]
$$

is the thrust vector expressed in $\mathbb{F}_{H}, T$ is the thrust vector magnitude, $\alpha_{B}$ is the lander's vertical attitude angle and $\psi_{B}$ is the lander's horizontal attitude angle.

The lander's mass flow rate equation is given as

$$
\dot{m}=-T /\left(I_{s p} g_{E}\right)
$$

where $I_{s p}$ is the lander's specific impulse and $g_{E}$ is the gravitational acceleration on the Earth's surface.

\subsection{Dimensionless Model}

In order to investigate closed form solutions, it is convenient to introduce dimensionless height, velocity, time and mass as

$$
\begin{aligned}
\tilde{h} & =\left(r-R_{m}\right) / R_{m}=h / R_{m} \\
\tilde{u} & =u / V_{m}, \tilde{v}=v / V_{m}, \tilde{w}=w / V_{m} \\
\tilde{t} & =\Omega_{m} t \\
\tilde{m} & =m / m_{0}
\end{aligned}
$$


where, $\mu$ is planetary gravitational constant, $R_{m}$ is planetary dimensional radius, $V_{m}=\sqrt{\mu / R_{m}}, \Omega_{m}=\sqrt{\mu / R_{m}^{3}}$, and $m_{0}$ is initial dimensional mass of the lander.

Now, Equation (1) can be transformed to a dimensionless form

$$
\begin{aligned}
\frac{d \tilde{h}}{d \tilde{t}} & =-\tilde{w} \\
\frac{d \phi}{d \tilde{t}} & =\frac{1}{1+\tilde{h}} \tilde{u} \\
\frac{d \lambda}{d \tilde{t}} & =\frac{1}{(1+\tilde{h}) \cos \phi} \tilde{v} \\
\frac{d \tilde{u}}{d \tilde{t}} & =-\frac{\tilde{v}^{2}}{1+\tilde{h}} \tan \phi+\frac{\tilde{u} \tilde{w}}{1+\tilde{h}}-\tilde{a}_{N} \cos \alpha_{B} \cos \psi_{B} \\
\frac{d \tilde{v}}{d \tilde{t}} & =\frac{\tilde{u} \tilde{v}}{1+\tilde{h}} \tan \phi+\frac{\tilde{v} \tilde{w}}{1+\tilde{h}}-\tilde{a}_{N} \cos \alpha_{B} \sin \psi_{B} \\
\frac{d \tilde{w}}{d \tilde{t}} & =-\frac{\tilde{u}^{2}+\tilde{v}^{2}}{1+\tilde{h}}+\frac{1}{(1+\tilde{h})^{2}}-\tilde{a}_{N} \sin \alpha_{B}
\end{aligned}
$$

where, $\tilde{a}_{N}=\tilde{a}_{N 0} / \tilde{m}$ and $\tilde{a}_{N 0}=T /\left(m_{0} g_{0}\right), g_{0}=\mu / R_{m}^{2}$. The dimensionless form of the mass equation (3) can be written as

$$
d \tilde{m} / d \tilde{t}=-p
$$

where, $p=T /\left(I_{s p} g_{E} \Omega_{m} m_{0}\right)$.

\section{CLOSED FORM OPTIMAL SOLUTION}

This paper is focused on the guidance law of planetary landing problem. The attitude control problem is not discussed in this paper. For the consideration of simplification but without losing integration, the soft landing process is divided into two phases. The first phase is initial braking phase. In this phase, the relative velocity of the spacecraft to planet will drop to $0 \mathrm{~m} / \mathrm{s}$. The most fuel is consumed in the initial braking phase. This phase is also referred to as powered descent phase in many literatures $[10,13]$. The second phase is gravity turn phase which assure lander to be vertical on the planetary surface. The guidance and control of gravity turn phase has been studied in Ref. [8], so it will not be discussed in detail here. The guidance and control of the initial braking phase is mainly studied in this paper. 
Consider an initial braking phase in which the landing point is not specified exactly, but rather the three dimensional velocity and height at the terminal point are specified, and the terminal dimensional time $t_{f}\left(\tilde{t}_{f}\right)$ is free [10]. The boundary conditions are specified as

$$
\begin{gathered}
h\left(t_{0}\right)=h_{0}, u\left(t_{0}\right)=u_{0}, v\left(t_{0}\right)=v_{0}, w\left(t_{0}\right)=w_{0} \\
h\left(t_{f}\right)=h_{f}, u\left(t_{f}\right)=u_{f}, v\left(t_{f}\right)=v_{f}, w\left(t_{f}\right)=w_{f}
\end{gathered}
$$

or in a dimensionless form using equation (4).

The objective is to find the optimal attitude angles $\psi_{B}$ and $\alpha_{B}$, which minimize fuel consumption and satisfy the terminal conditions. Using optimal control theory, the optimal steering law is a linear tangent law $[10,12]$

$$
\begin{aligned}
\tan \psi_{B} & =\tilde{a} \\
\tan \alpha_{B} & =\tilde{c} \tilde{t}+\tilde{d} \equiv \tilde{\tau}
\end{aligned}
$$

where $\tilde{a}, \tilde{c}$ and $\tilde{d}$ are unknown constants given by solving a two point boundary value problem. The constant $\tilde{a}$ can be solved from the boundary conditions as

$$
\tilde{a}=\frac{\tilde{v}\left(\tilde{t}_{f}\right)-\tilde{v}(0)}{\tilde{u}\left(\tilde{t}_{f}\right)-\tilde{u}(0)}
$$

A numerically optimal solution can then be generated by solving the two-point boundary problem. However, in general, closed form solutions for the other two coefficients form cannot be found.

To find a closed form solution, small terms $o(1)$ in equation (5) are omitted, which are $\tilde{u} \tilde{w} /(1+\tilde{h})$ and $\tilde{v} \tilde{w} /(1+\tilde{h})$. In addition, $u_{0}$ is assumed to be much larger than other velocities in boundary conditions. This assumption has been used in Ref. [10]. Therefore the terms of $\tilde{v}^{2} \tan \phi /(1+\tilde{h})$ and $\tilde{u} \tilde{v} \tan \phi /(1+\tilde{h})$ can also be omitted as small terms. Substituting the optimal angles equation (7) into equation (5), the equations of motion now reduce to

$$
\begin{aligned}
\frac{d \tilde{h}}{d \tilde{t}} & =-\tilde{w} \\
\frac{d \tilde{u}}{d \tilde{t}} & =-\tilde{a}_{N} \frac{1}{\sqrt{1+\tilde{a}^{2}}} \frac{1}{\sqrt{1+\tilde{\tau}^{2}}} \\
\frac{d \tilde{v}}{d \tilde{t}} & =-\tilde{a}_{N} \frac{\tilde{a}}{\sqrt{1+\tilde{a}^{2}}} \frac{1}{\sqrt{1+\tilde{\tau}^{2}}} \\
\frac{d \tilde{w}}{d \tilde{t}} & =-\frac{\left(\tilde{u}^{2}+\tilde{v}^{2}\right)}{1+\tilde{h}}-\tilde{a}_{N} \frac{\tilde{\tau}}{\sqrt{1+\tilde{\tau}^{2}}}+\frac{1}{(1+\tilde{h})^{2}}
\end{aligned}
$$


where, $\tilde{a}_{N}=\tilde{a}_{N 0} /(1-p \tilde{t})$.

In the initial braking phase, the vehicle attitude is mostly horizontal [14], thus $|\tilde{\tau}|<1$. In addition, $\tilde{h} \ll 1$ and $p \tilde{t}$ is assumed to be smaller than 1 . This assumption can be also verified using data from references $[9-11,13]$. Therefore the thrust and gravitational acceleration, and the cosine of the vertical attitude angle are expanded into the following polynomials,

$$
\begin{aligned}
1 /(1-p \tilde{t}) & \cong \varsigma=1+p \tilde{t}+p^{2} \tilde{t}^{2}+p^{3} \tilde{t}^{3}+p^{4} \tilde{t}^{4}+p^{5} \tilde{t}^{5} \\
1 /(1+\tilde{h})^{2} & \cong 1-2 \tilde{h} \\
1 / \sqrt{1+\tilde{\tau}^{2}} & \cong f=1-0.5 \tilde{\tau}^{2}+0.375 \tilde{\tau}^{4}-0.3125 \tilde{\tau}^{6}
\end{aligned}
$$

At the beginning of the initial braking phase, the spacecraft is traveling at a speed fairly close to $V_{m}$ and its attitude is almost horizontal. Therefore, $\left(\tilde{u}^{2}+\tilde{v}^{2}\right) /(1+\tilde{h})$ cannot be omitted as a higher order term in equation (9). However, $\left(\tilde{u}^{2}+\tilde{v}^{2}\right) /(1+\tilde{h}) \rightarrow o(1)$ as $\tilde{t} \rightarrow \tilde{t}_{f}$, thus we replace $\tilde{h}$ with $\tilde{h}_{0}$ in the term of $\left(\tilde{u}^{2}+\tilde{v}^{2}\right) /(1+\tilde{h})$. Equation (9) can then be simplified as,

$$
\begin{aligned}
\frac{d \tilde{h}}{d \tilde{t}} & =-\tilde{w} \\
\frac{d \tilde{u}}{d \tilde{t}} & =-\tilde{a}_{N 0} \frac{1}{\sqrt{1+\tilde{a}^{2}}} \varsigma f \\
\frac{d \tilde{v}}{d \tilde{t}} & =-\tilde{a}_{N 0} \frac{\tilde{a}}{\sqrt{1+\tilde{a}^{2}}} \varsigma f \\
\frac{d \tilde{w}}{d \tilde{t}} & =-\frac{\left(\tilde{u}^{2}+\tilde{v}^{2}\right)}{1+\tilde{h}_{0}}-\tilde{a}_{N 0} \varsigma f \tilde{\tau}+1-2 \tilde{h}
\end{aligned}
$$

Substituting equation (8) into equation (11b) and equation (11c), the horizontal velocities $\tilde{u}(\tilde{t})$ and $\tilde{v}(\tilde{t})$ can be solved using the initial condition $\tilde{u}_{0}$ and $\tilde{v}_{0}$. The solution for the horizontal velocity $\tilde{u}(\tilde{t})$ can be written as

$$
\tilde{u}(\tilde{t})=\sum_{i=0}^{n} \tilde{u}_{i} \tilde{t}^{i}
$$

where $\tilde{u}_{i}(\tilde{c}, \tilde{d})(i>0)$ are unknown constants determined by $\tilde{c}$ and $\tilde{d}$. The horizontal velocity $\tilde{v}(\tilde{t})$, related with $\tilde{u}(\tilde{t})$, can be written as follows,

$$
\tilde{v}(\tilde{t})=\tilde{a} \tilde{u}(\tilde{t})
$$

Substituting equation (12) into equation (11d) and equation (11a), the following 
altitude equation is derived

$$
\tilde{h}^{\prime \prime}(\tilde{t})=q(\tilde{t})+2 \tilde{h}(\tilde{t})
$$

where,

$$
\begin{aligned}
q(\tilde{t}) & =\frac{\left(\tilde{u}(\tilde{t})^{2}+\tilde{v}(\tilde{t})^{2}\right)}{1+\tilde{h}_{0}}+\tilde{a}_{N 0} \varsigma f \tau-1 \\
& \cong \sum_{i=0}^{m} q_{i} \tilde{t}^{i}
\end{aligned}
$$

It is still not possible to solve for the vertical velocity and height from equation (14) in closed forms. However, the vertical velocity can be solved as a polynomial if the gravitational acceleration is assumed to be constant in equation (14). Note that $\tilde{h}(\tilde{t}) \ll 1$, so it is reasonable to assume $\tilde{w}(\tilde{t})$ and $\tilde{h}(\tilde{t})$ are polynomial functions of time even if the variation of gravitational acceleration with height is considered. Therefore, the following polynomial iteration method can be used to obtain $\tilde{h}(\tilde{t})$ and $\tilde{w}(\tilde{t})$. Suppose,

$$
\tilde{h}(\tilde{t})=\sum_{i=0}^{n} \tilde{h}_{i} \tilde{t}^{i}
$$

is the approximate solution of equation (14), where $n=m+2$ and $\tilde{h}_{i}$ are unknown constants. Substitute equation (16) into equation (14), so that the coefficients of equation (16) can be solved using the initial conditions $\tilde{h}_{0}$ and $\tilde{h}_{1}=-\tilde{w}_{0}$, from the following equations,

$$
\begin{aligned}
\tilde{h}_{2} & =\left(q_{0}+2 \tilde{h}_{0}\right) / 2 \\
\tilde{h}_{3} & =\left(q_{1}+2 \tilde{h}_{1}\right) / 6 \\
\ldots & \\
\tilde{h}_{n-1} & =\left(q_{m-1}+2 \tilde{h}_{m-1}\right) /((m-2)(m-1)) \\
\tilde{h}_{n} & =\left(q_{m}+2 \tilde{h}_{m}\right) /((m-1) m)
\end{aligned}
$$

Thereafter, height and vertical velocity can be written as

$$
\begin{aligned}
& \tilde{h}(\tilde{t})=\sum_{i=0}^{n} \tilde{h}_{i} \tilde{t}^{i} \\
& \tilde{w}(\tilde{t})=\sum_{i=0}^{n-1} \tilde{w}_{i} \tilde{t}^{i}
\end{aligned}
$$


where $\tilde{h}_{i}(\tilde{c}, \tilde{d})(i>0)$ and $\tilde{w}_{i}(\tilde{c}, \tilde{d})(i>0)$ are unknown constants determined by $\tilde{c}, \tilde{d}$.

Substituting the terminal condition of $\tilde{u}, \tilde{v}, \tilde{w}$ and $\tilde{h}$ into algebraic equations (12), (13) and (18), the four unknown constants $\tilde{a}, \tilde{c}, \tilde{d}$, and $\tilde{t}_{f}$ can be determined analytically from four terminal conditions. Therefore, a closed form optimal solution is obtained.

Compared to other analytical approaches of this guidance law, the variations of lander's mass and gravitational acceleration are considered to solve the analytical solution in this paper. Therefore, it is a good approximation for numerical solution which will be verified in subsection 5.1. In addition, because the mentioned strategy is in the analytic solution, several difficulties associated with the numerical determination of optimal control solution for nonlinear systems, such as slow convergence rate and high sensitivity to initial guesstimates, do not appear [11].

\section{CONTROL}

With the closed form analytic solution obtained, the next step is to develop control laws which track this reference trajectory. The thruster force $T$, vertical attitude angle $\alpha_{B}$, and horizontal attitude angle $\psi_{B}$ are used to track the reference height, reference vertical velocity and reference horizontal velocity. In the initial braking phase, a velocity error and height error are defined as,

$$
\begin{aligned}
e_{u}(t) & =u(t)-u_{d}(t) \\
e_{v}(t) & =v(t)-v_{d}(t) \\
e_{w}(t) & =w_{d}(t)-w(t) \\
e_{h}(t) & =h(t)-h_{d}(t)
\end{aligned}
$$

and the transformed control signals are introduced as

$$
\begin{aligned}
& u_{u}=T \cos \psi_{B} \cos \alpha_{B}-T_{d} \cos \psi_{B d} \cos \alpha_{B d} \\
& u_{v}=T \sin \psi_{B} \cos \alpha_{B}-T_{d} \sin \psi_{B d} \cos \alpha_{B d}
\end{aligned}
$$


where $u_{d}(t), v_{d}(t), w_{d}(t), h_{d}(t)$ are closed form solutions generated from (12) and (18), $\psi_{B d}$ and $\alpha_{B d}$ are the optimal attitude angles derived from (7), and $T_{d}$ is the nominal thrust force.

Using definitions (19a) and (19b) with equation (1), the error dynamics of the horizontal velocity can be written as,

$$
\begin{aligned}
& \dot{e}_{u}=-\frac{1}{m} u_{u}+d_{u} \\
& \dot{e}_{v}=-\frac{1}{m} u_{v}+d_{v}
\end{aligned}
$$

where $d_{u}$ and $d_{v}$ are nonlinear terms. Obviously, the system (21) is controllable, since the rank of controllability matrix is two.

Denoting $e_{h w}(t)=\left[e_{h}(t), e_{w}(t)\right]^{\top}$, using definitions (19d) and (19c) with equation (1), the error dynamics of the lander altitude can be written as,

$$
\dot{e}_{h w}(t)=A_{h w} e_{h w}(t)+B_{h w} u_{h w}+d_{h w}
$$

where

$$
A_{h w}=\left[\begin{array}{cc}
0 & 1 \\
\frac{2 g_{0}}{R_{m}} & 0
\end{array}\right] \quad B_{h w}=\left[\begin{array}{c}
0 \\
\frac{1}{m}
\end{array}\right]
$$

, and

$$
u_{h w}=T \sin \alpha_{B}-T_{d} \sin \alpha_{B d}
$$

is the transformed control signal for height system, and $d_{h w}$ is the higher-order infinitesimal of $e_{h w}: o\left(e_{h w}^{2}\right)$. Obviously, the system (22) is also controllable, since the rank of controllability matrix is two.

\subsection{Fixed Gain Control}

To design a FGC law, the nonlinear terms in equations (21) and (22) are omitted.

The feedback control signals can then be written as

$$
\begin{aligned}
u_{u} & =K_{u} e_{u} \\
u_{v} & =K_{v} e_{v} \\
u_{h w} & =K e
\end{aligned}
$$


where, $K=\left[K_{h}, K_{w}\right]$.

Neglecting the variation of the lander's mass, the closed loop poles of the systems (21) and (22) can be determined as

$$
\begin{aligned}
s_{u} & =-\frac{K_{u}}{m} \\
s_{v} & =-\frac{K_{v}}{m} \\
s_{h w} & =-\xi \omega_{n} \pm \omega_{n} \sqrt{\xi^{2}-1}
\end{aligned}
$$

where $s_{u}, s_{v}$ are the closed-loop poles of (21), $s_{h w}$ are the closed-loop poles of $(22)$ and

$$
\begin{aligned}
\xi & =\frac{-K_{w} / m}{\sqrt{-\left(2 g_{0} / R_{m}+K_{h} / m\right)}} \\
\omega_{n} & =\sqrt{-\left(2 g_{0} / R_{m}+K_{h} / m\right)}
\end{aligned}
$$

Using linear system theory, it can be shown that $t \rightarrow \infty e_{u} \rightarrow 0, e_{v} \rightarrow 0, e \rightarrow 0$, if,

$$
\begin{aligned}
K_{u}, K_{v} & >0 \\
K_{h} & <-\frac{2 m_{0} g}{R_{m}} \\
K_{w} & <0
\end{aligned}
$$

\subsection{Direct Adaptive Control}

In Ref. [8], a DAC law is designed for gravity turn guidance to compensate for un-modeled dynamics and to cope with thruster failure during the terminal descent maneuver. It will be demonstrated in this section, that this control law can also be used to track height and vertical velocity in initial braking phase.

Theorem 1 Consider the following first-order linear system:

$$
\dot{x}(t)=a x(t)+b u(t)+d
$$

where $a, b$, and $d \in \Re$ are assumed to be unknown except for the sign of $b$, which is assumed to be negative. A control law is now required which has the property that $x(t) \rightarrow 0$ as $t \rightarrow \infty$ without knowledge of $a, b$ and $d$. It can be shown that 
the control law

$$
u(t)=k_{1}(t) x(t)+k_{2}(t)
$$

possesses these properties, where the control gains are obtained from

$$
\begin{aligned}
& \dot{k_{1}}(t)=\tau_{1} x^{2}(t) \\
& \dot{k_{2}}(t)=\tau_{2} x(t)
\end{aligned}
$$

with the parameters $\tau_{1}, \tau_{2}>0$.

A detailed proof of this theorem is presented in Ref. 15 or Ref. 16 .

Theorem 2 Consider the linear system

$$
\dot{x}(t)=A x(t)+B u(t)+d
$$

where

$$
A=\left[\begin{array}{c}
A_{0} \\
a
\end{array}\right], B=\left[\begin{array}{l}
0 \\
b
\end{array}\right], d=\left[\begin{array}{c}
0 \\
d_{0}
\end{array}\right]
$$

and $x(t)=\left[x_{1}(t), x_{2}(t)\right]^{T}, u(t) \in \Re, d \in \Re^{2}, A_{0} \in \Re^{1 \times 2}, a \in \Re^{1 \times 2}, b, d_{0} \in \Re$ and $b>0$. A control law now is required which has the property that $x(t) \rightarrow 0$ as $t \rightarrow \infty$ without knowledge of $a, b, d_{0}$ except for the sign of $b$. It can be shown that the control law

$$
u(t)=K_{1}(t) x_{1}(t)+K_{2}(t) x_{2}(t)+K_{3}(t)
$$

possesses these properties, where the control gains are obtained from

$$
\begin{aligned}
\dot{K}_{1}(t) & =-\lambda_{1}\left[p x_{1}^{2}(t)+x_{1}(t) x_{2}(t)\right] \\
\dot{K}_{2}(t) & =-\lambda_{2}\left[x_{2}^{2}(t)+p x_{1}(t) x_{2}(t)\right] \\
\dot{K}_{3}(t) & =-\lambda_{3}\left[p x_{1}^{2}(t)+x_{2}(t)\right]
\end{aligned}
$$

with the parameters $\lambda_{i}(i=1,2,3), p>0$.

A detailed proof of this theorem is presented in Ref. [17], using Lyapunov methods. More theoretical and practical issues can be found in Ref. $[18,19]$. 
It can be seen that (21) is of the form of (29). Using Theorem 1, the control law to track horizontal velocity can be written as

$$
\begin{aligned}
& u_{u}=k_{1}(t) e_{u}(t)+k_{2}(t) \\
& u_{v}=k_{3}(t) e_{v}(t)+k_{4}(t)
\end{aligned}
$$

where the update equation of gains are obtained from

$$
\begin{aligned}
& \dot{k}_{1}(t)=\tau_{1} e_{u}^{2}(t) \\
& \dot{k}_{2}(t)=\tau_{2} e_{u}(t) \\
& \dot{k}_{3}(t)=\tau_{3} e_{v}^{2}(t) \\
& \dot{k}_{4}(t)=\tau_{4} e_{v}(t)
\end{aligned}
$$

with the constants $\tau_{i}>0(i=1,2,3,4)$.

Equation (22) is of the form of (33), with nonlinear terms grouped as an unknown disturbance. Using Theorem 2, the control law for tracking height can be written as

$$
u_{h w}=K_{1}(t) e_{h}(t)+K_{2}(t) e_{w}(t)+K_{3}(t)
$$

where the gains are obtained from

$$
\begin{aligned}
\dot{K}_{1}(t) & =-\lambda_{1}\left[p e_{h}^{2}(t)+e_{h}(t) e_{w}(t)\right] \\
\dot{K}_{2}(t) & =-\lambda_{2}\left[e_{w}^{2}(t)+p e_{h}(t) e_{w}(t)\right] \\
\dot{K}_{3}(t) & =-\lambda_{3}\left[p e_{h}^{2}(t)+e_{w}(t)\right]
\end{aligned}
$$

with the constants $\lambda_{i}(i=1,2,3), p>0$.

The control variables $T, \alpha_{B}$ and $\psi_{B}$ can be calculated from (20) and (24).

\section{EXAMPLE MISSION AND NUMERICAL RE- SULTS}

In this section, a numerical simulation of lunar soft landing mission is implemented to validate the closed form solution and evaluate the performance of the 
Table 1 Lunar constants

\begin{tabular}{lc}
\hline Name & Value \\
\hline$\mu$ & $4.9028 \times 10^{12} \mathrm{~m}^{3} / \mathrm{s}^{2}$ \\
$R_{m}$ & $1.738 \times 10^{6} \mathrm{~m}$ \\
$V_{m}$ & $1.6796 \times 10^{3} \mathrm{~m} / \mathrm{s}$ \\
$\Omega_{m}$ & $9.6638 \times 10^{-4} \mathrm{rad} / \mathrm{s}$ \\
$g_{0}$ & $1.6231 \mathrm{~m} / \mathrm{s}^{2}$ \\
\hline
\end{tabular}

control laws presented in the preceding section. A sample soft landing typical of a Lunar Cargo Transportation system [20] is produced. The initial braking phase is from $15 \mathrm{~km}$ to $100 \mathrm{~m}$ altitude. The analytical optimal trajectory based on the linear tangent guidance law in Sec. 3 is used. The velocity of lander will also reduces to zero in this phase. The second phase is from $100 \mathrm{~m}$ to lunar surface. During this phase, gravity-turn guidance is used to track a velocity-height profile which assure lander's vertical attitude on the lunar surface. More details of descent profile in the gravity-turn phase can be found in Ref. [8].

Values of the lunar constants in equation (4) are shown in Table 1. Boundary conditions for the initial braking phase are given as follows

$$
\begin{aligned}
& h_{0}=15 \mathrm{~km}, u_{0}=1609.08 \mathrm{~m} / \mathrm{s}, v_{0}=100 \mathrm{~m} / \mathrm{s}, w_{0}=0 \\
& h_{f}=100 \mathrm{~m}, u_{f}=0, v_{f}=0, w_{0}=0
\end{aligned}
$$

In addition, the lander's velocity on the lunar surface should be lower than $0.5 \mathrm{~m} / \mathrm{s}$. The assumed initial mass is $5156 \mathrm{~kg}$, the nominal thruster force is $24 \mathrm{kN}$ with a thruster $I_{s p}=315 \mathrm{~s}$, and a $30 \%$ margin on thrust is provided.

\subsection{Closed Form Optimal Solution vs Numerical Optimal Solution}

The unknown parameter $\tilde{a}$ is solved from equation (8) as $\tilde{a}=0.0621$. Substituting the boundary conditions equation (41) into algebraic equations (12) and (18), the other three unknown constants parameters $\tilde{c}, \tilde{d}$ and $\tilde{t}_{f}$ can be specified 


$$
\tilde{c}=2.2599, \tilde{d}=-0.1413, \tilde{t}_{f}=0.2660
$$

The closed form optimal solution can be then obtained from equation (12) and equation (18). To examine the accuracy of closed form solution, the numerical optimal solution can be obtained using steepest descent method [11]. The values of unknown constants for numerical results are

$$
\tilde{a}=0.0632, \tilde{c}=2.2297, \tilde{d}=-0.1388, \tilde{t}_{f}=0.2676
$$

Both the closed form solution and numerical solution are shown in Fig. 2. It can be seen that the closed form solution provides an extremely good match to the numerical solution, and as such it can be used as an optimal reference trajectory.

\subsection{Fixed Gain Control vs Direct Adaptive Control}

The closed form solution obtained earlier is used to define a reference trajectory. To show the validity of the control laws developed, a limited monte-carlo campaign through a range of initial condition errors is operated. The mean errors are zero, the standard deviations are shown in Table 2. To yield desirable closed-loop performance in terms of both transient and steady state response characteristics, the overshoot is chosen less than $10 \%$ and rise time is chosen less than $50 \mathrm{~s}$. The closed-loop poles of systems (21) and (22) can then be determined [21] and theoretical values of FGC gains can be calculated from (26). Based on theoretical values, actual values of FGC gains are then tuned which are shown in Table 3. The values of the DAC law parameters are shown in Table 4 and Table 5. Both FGC and DAC developed in Sec. 4 are able to track the reference trajectory. Numerical results of the monte-carlo simulation are shown in Fig. 3. The lander has a $98.9 \%$ probability to land in the area of the ellipse, which is a $3-\sigma$ landing ellipse within the target range. Next, the thruster failure situation is considered to analyze the impact velocities and evaluate the displacement of the landing site. 


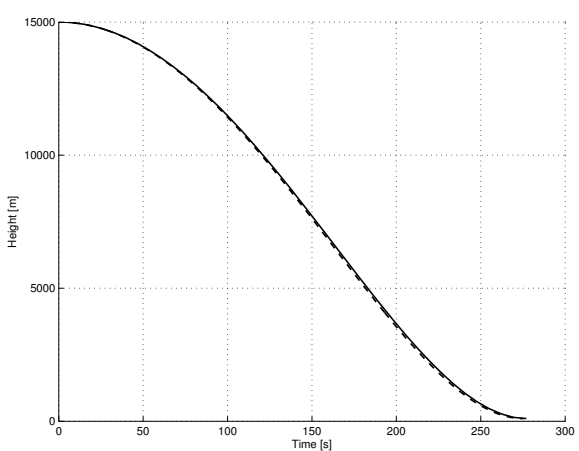

(a) Height vs time

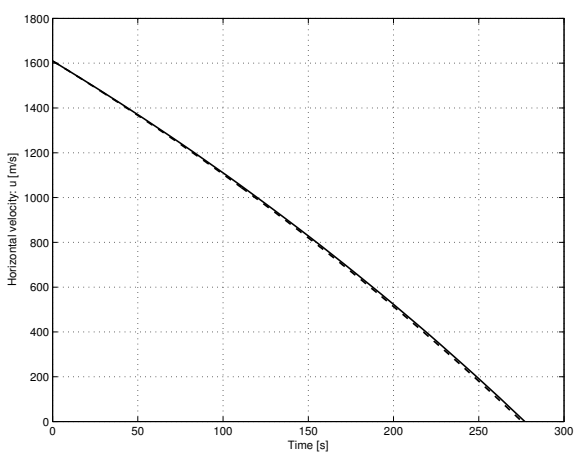

(c) Horizontal velocity: u vs time

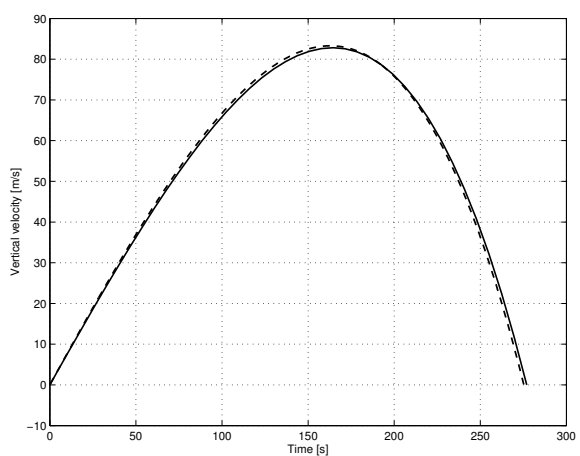

(b) Vertical velocity: w vs time

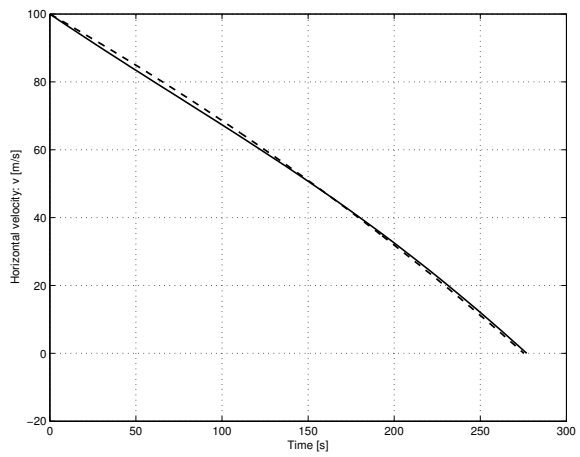

(d) Horizontal velocity: v vs time

Fig. 2 Comparing analytical solution with numerical solution.(--, closed form solution; -, numerical solution)

Table 2 Values of initial condition errors for monte-carlo simulation

\begin{tabular}{lc}
\hline Variable & Standard deviation \\
\hline position error & $300 \mathrm{~m}$ \\
horizontal velocity error & $2.5 \mathrm{~m} / \mathrm{s}$ \\
vertical velocity error & $5 \mathrm{~m} / \mathrm{s}$ \\
\hline
\end{tabular}




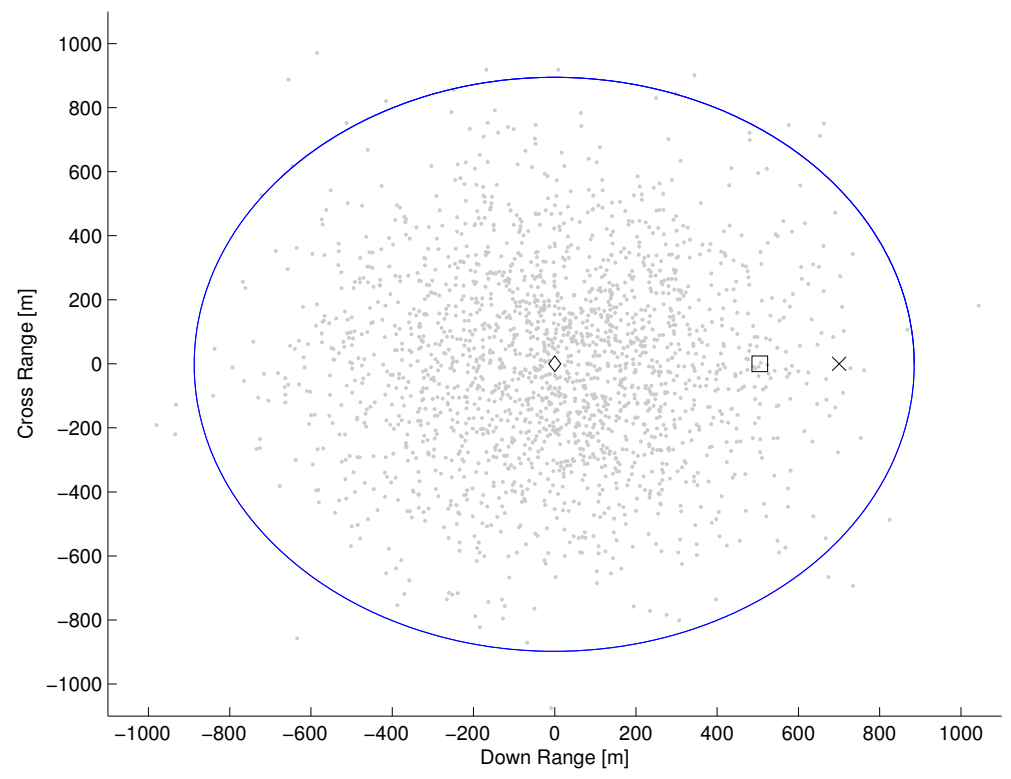

Fig. 3 Landing sites of Monte-carlo simulation result.(Diamond, nominal site; Square, DAC result with $30 \%$ thrust failure; X-mark, FGC result $(\delta(t)=0 \mathrm{~s})$ with $30 \%$ of thrust failure)

For actuator failure compensation, a certain redundancy of actuators is needed [22]. As the engine is running at $70 \%$ maximum available thrust in the nominal scenario, the failure condition is to fail the engine performance by $30 \%$ at the midpoint of the descent maneuver. This failure mode is modeled by multiplying the commanded thrust by [8].

$$
f(t)=\frac{1+T_{a}}{2}\left[1-\frac{2}{\pi} \frac{1-T_{a}}{1+T_{a}} \tan ^{-1}\left(2 t-t_{f}\right)\right]
$$

with $T_{a}=0.7$. In addition, based on Table 2 , the initial height error is set to $300 \mathrm{~m}$ and initial velocity error is set to $[2.5,3,5]^{\top} \mathrm{m} / \mathrm{s}$. If there is no fault detection to detect thruster failure, the normal gains are used throughout the mission for FGC, whereas DAC can adjust its gains. The values of the control parameters are also shown in Table 3, Table 4 and Table 5. Numerical results and control variables in optimal phase are shown in Fig. 4 and Fig. 5. As shown from the simulation results, at the beginning of simulation, there is a tracking error due to the initial conditions, and this tracking error goes to zero as time elapses for FGC and DAC. Therefore, FGC and DAC are able to track 
Table 3 FGC control parameters in optimal phase

\begin{tabular}{lc}
\hline Case & FGC parameters \\
\hline Normal Gains & $K_{u}=50, K_{v}=400, K_{w}=-80, K_{h}=-5$ \\
Backup Gains & $K_{u}=2000, K_{v}=2000, K_{w}=-500, K_{h}=-500$ \\
\hline
\end{tabular}

Table 4 DAC control parameters in optimal phase

\begin{tabular}{lc}
\hline Case & DAC parameters \\
\hline adaptation weights $^{\dagger}$ & $\tau_{1}=14.5, \tau_{2}=1, \tau_{3}=100, \tau_{4}=1$ \\
adaptation weights ${ }^{\ddagger}$ & $\lambda_{1}=0.005, \lambda_{2}=0.5, \lambda_{3}=0.001, p=0.1$ \\
initial conditions of gains ${ }^{\dagger}$ & $k_{1}(0)=-100, k_{2}(0)=0, k_{3}(0)=100, k_{4}(0)=100$ \\
initial conditions of gains ${ }^{\ddagger}$ & $K_{1}(0)=1, K_{2}(0)=1, K_{3}(0)=1$ \\
\hline$\dagger$ track horizontal velocity & \\
$\ddagger$ track height system &
\end{tabular}

the reference trajectory asymptotically initially. When thrust failures occur, there is a transient response in the errors of height and velocity. For DAC, the gains are thus to be changed from equation (38) and (40) to compensate for the thrust failures, and the actual values of control variables $\left(T, \alpha_{B}\right.$ and $\left.\psi_{B}\right)$ can still be controlled to around the reference values after transient response (see Fig. 5). Therefore, DAC can still track the reference trajectory by adjusting its gains automatically because of embedded autonomy. FGC, however, is unable to further track the reference trajectory. Compared to FGC, DAC has the ability to handle thrust failure and is thus more robust, enabling fault detection, isolation and recovery (FDIR) by the control system and eliminating the need for active fault detection.

Next, another situation is considered. Now a fault detection algorithm is assumed, so the gains are switched to a set of backup values for FGC after thruster failure occurs. This would be the failure design limit. Thruster failure is typically detected through on-board monitoring of house-keeping data [23]; the 
Table 5 Control parameters in gravity-turn phase ${ }^{\dagger}$

\begin{tabular}{lc}
\hline Case & parameters \\
\hline DAC & $k_{1}(0)=20, k_{2}(0)=0, \tau_{1}=20 ; \tau_{2}=10$ \\
FGC normal gains & $k_{1}=10, k_{2}=0, \tau_{1}=0 ; \tau_{2}=0$ \\
FGC backup gains & $k_{1}=100, k_{2}=0, \tau_{1}=0 ; \tau_{2}=0$ \\
\hline
\end{tabular}

$\dagger$ meanings of these parameters can be found in Ref. 8

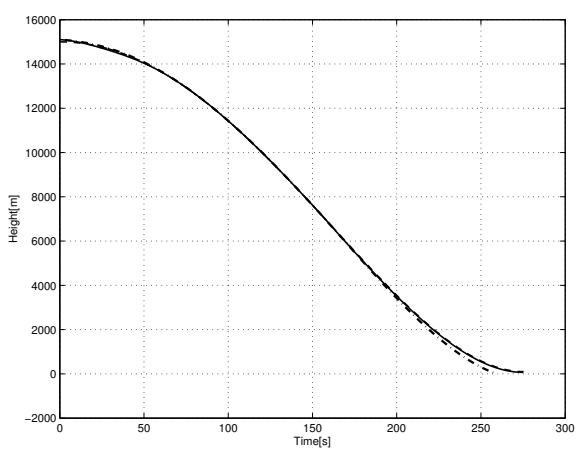

(a) Height vs time

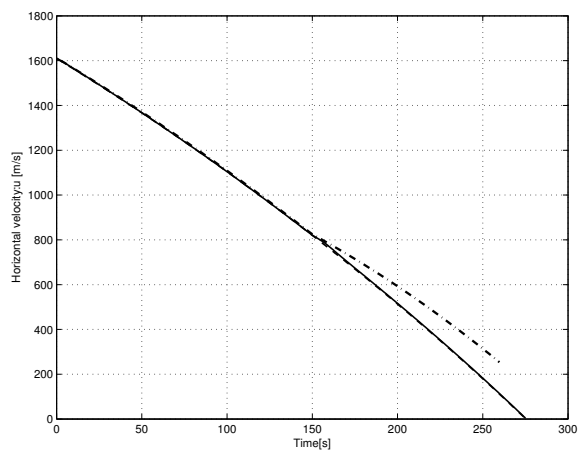

(c) Horizontal velocity: $\mathrm{u}(\mathrm{t})$ vs time

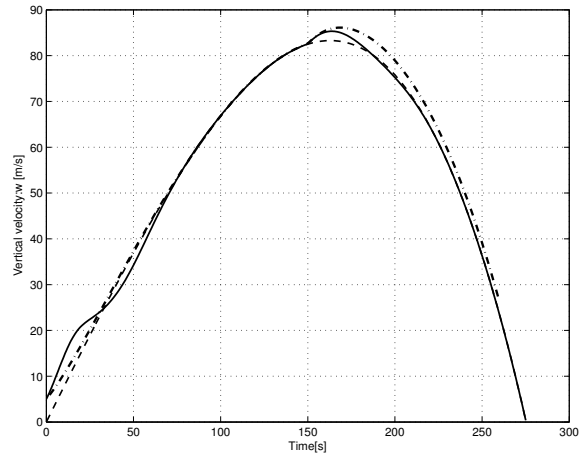

(b) Vertical velocity: $\mathrm{w}(\mathrm{t})$ vs time

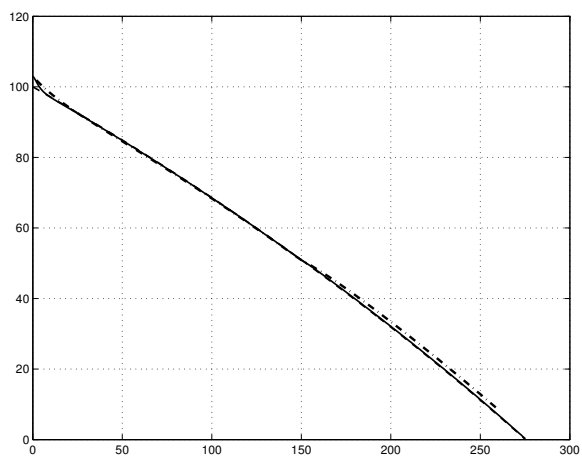

(d) Horizontal velocity: v(t) vs time

Fig. 4 Numerical results of optimal phase in the case of $30 \%$ thruster failure at the midpoint(--, required profile; -., FGC with normal gains; -, DAC) 


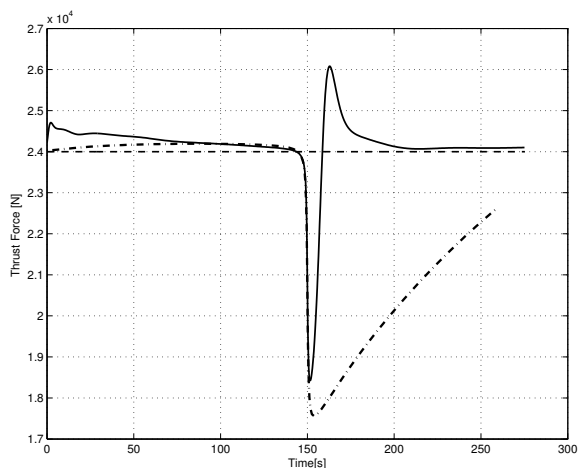

(a) Thruster force vs time

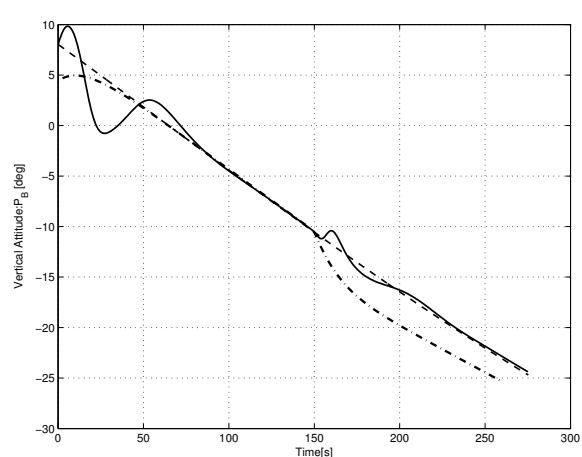

(b) Vertical attitude angle vs time

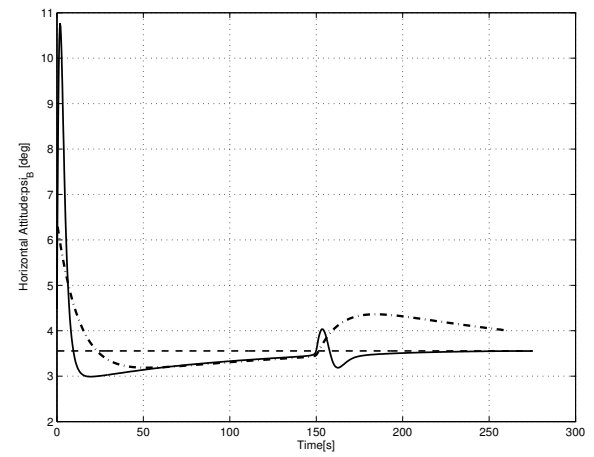

(c) Horizontal attitude angle vs time

Fig. 5 Control variables of optimal phase in the case of $30 \%$ thruster failure at the midpoint (--, required profile; -., FGC with normal gains; -, DAC) 
Table 6 Terminal velocity with $30 \%$ of thruster failed

\begin{tabular}{lc}
\hline Case & Terminal velocity \\
\hline DAC & {$[0,0,0.235]^{\top} \mathrm{m} / \mathrm{s}$} \\
FGC $(\delta(t)=0 \mathrm{~s})$ & {$[0,0,0.289]^{\top} \mathrm{m} / \mathrm{s}$} \\
FGC $(\delta(t)=1 \mathrm{~s})$ & {$[0,0,0.314]^{\top} \mathrm{m} / \mathrm{s}$} \\
FGC $(\delta(t)=5 \mathrm{~s})$ & {$[0,0,0.321]^{\top} \mathrm{m} / \mathrm{s}$} \\
\hline
\end{tabular}

Packet Utilization Standard (PUS) 12 Function Management Service system simply looks for frozen data or some other prolonged error and hence won't declare a fault until the error is detected on a predetermined number of consecutive cycles. Therefore, when using FGC, the Fault Detection, Isolation and Recovery (FDIR) system will not immediately switch to the back-up gains, rather this switch will be delayed by $\delta(t)$ seconds. The switch should thus be similarly delayed such that we are using the nominal, and wrong, control gains for $\delta(t)$ seconds. Numerical results for different values of $\delta(t)$ and control variables in the optimal phase are shown in Fig. 6 and Fig. 7. Numerical results and control variables for gravity turn are shown in Fig. 8. The terminal velocity at the lunar surface is shown in Table 6. It is shown that the tracking error converges to small values after a transient response caused by thruster failures. Therefore, the desired control system performance for FGC is also verified. The landing sites are shown in Fig. 3. It can be seen that both FGC and DAC are able to assure landing in the $3-\sigma$ ellipse. The actual landing site is $729.6 \mathrm{~m}$ far from the nominal site for FGC, whereas is $535.6 \mathrm{~m}$ far from the nominal site for DAC. Therefore, the DAC controller minimizes the error from the nominal landing site.

Compared to DAC shown in Fig. 4 and Fig. 5, FGC takes longer to adjust its attitude after thruster failure occurs. It is interesting to investigate a situation when thruster failure occurs near the terminal time of landing (i.e.,289 s). Numerical results for this scenario are shown in Fig 9, where the terminal ve- 


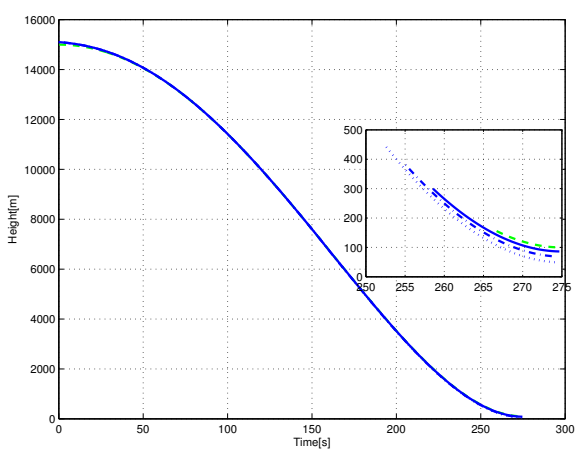

(a) Height vs time

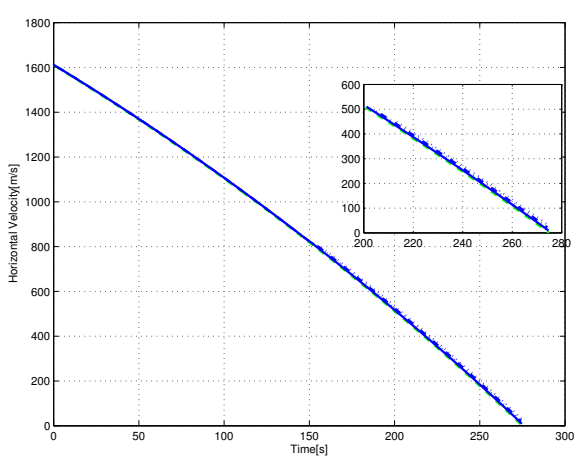

(c) Horizontal velocity:u(t) vs time

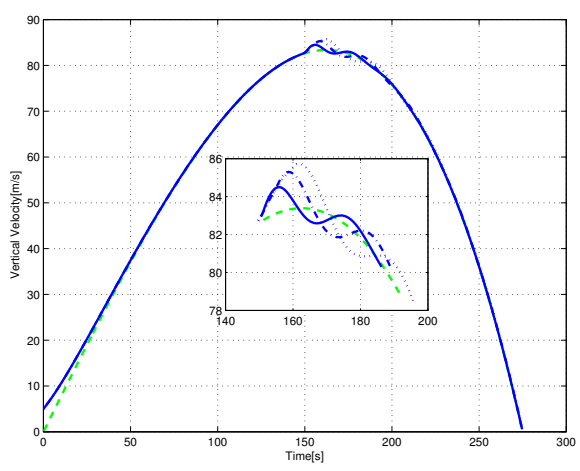

(b) Vertical velocity: $\mathrm{w}(\mathrm{t})$ vs time

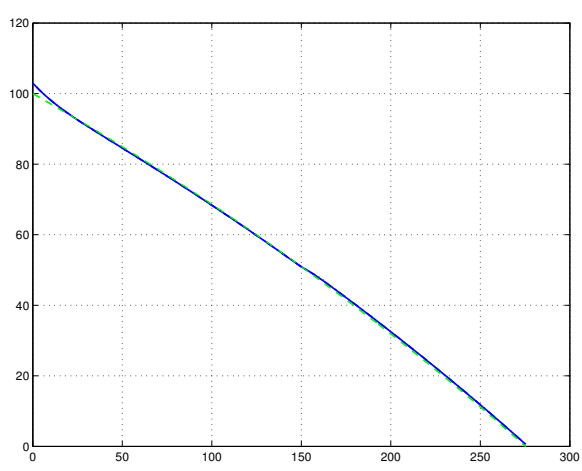

(d) Horizontal velocity:v(t) vs time

Fig. 6 Numerical results for FGC in optimal phase with different values of $\delta(t)$ after thruster failed (--, required profile; -, $\delta(t)=0 \mathrm{~s} ;-., \delta(t)=1 \mathrm{~s} ; \ldots, \delta(t)=5$ s ) 


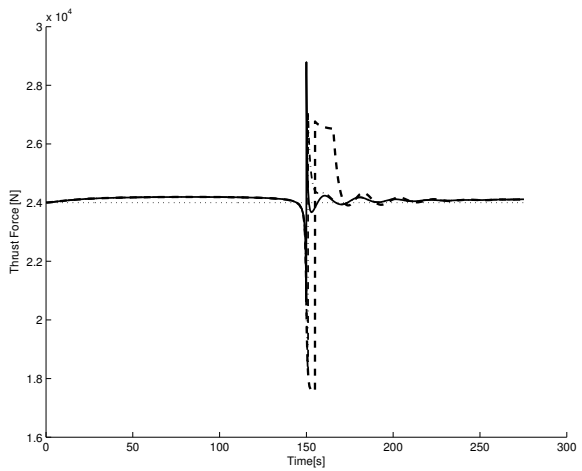

(a) Thruster force vs time

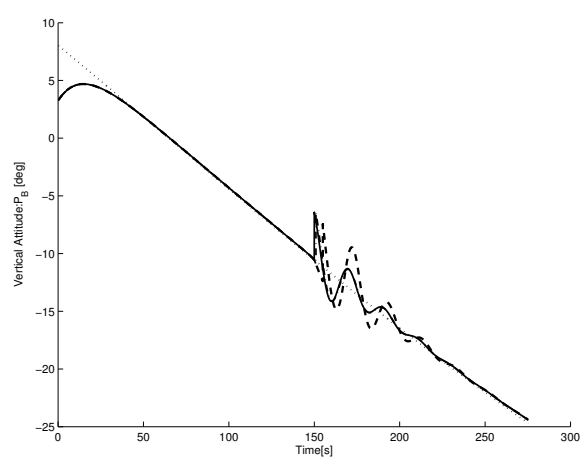

(b) Vertical attitude angle vs time

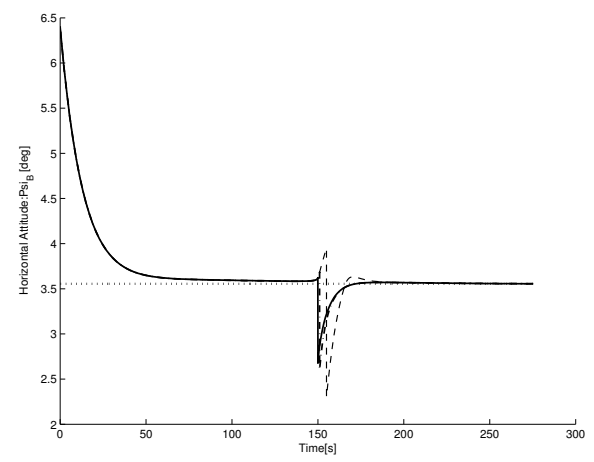

(c) Horizontal attitude angle vs time

Fig. 7 Control variables for FGC in optimal phase with different values of $\delta(t)$ after thruster failed $(\ldots$, required profile;,$- \delta(t)=0 \mathrm{~s} ;-., \delta(t)=1 \mathrm{~s} ;--, \delta(t)=5$ s ) 


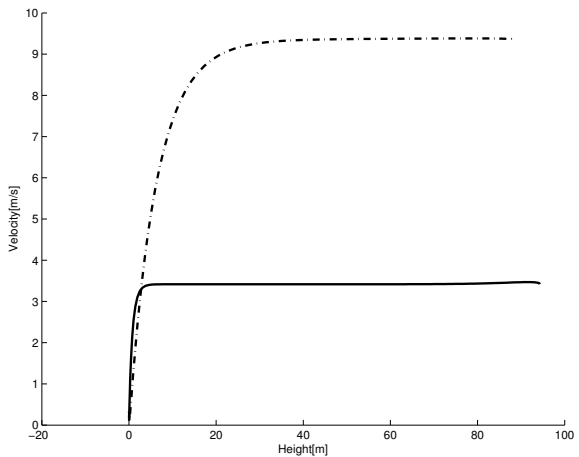

(a) Velocity vs height

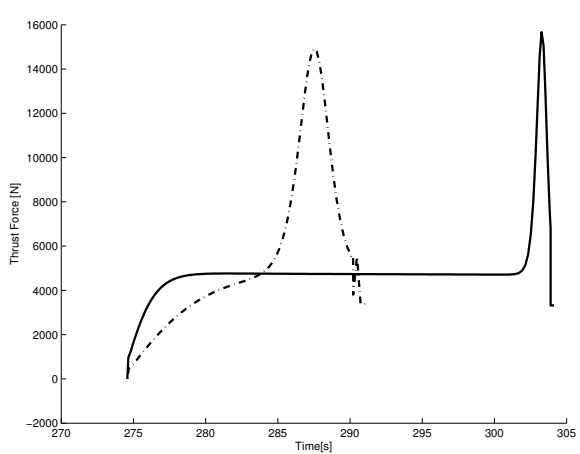

(b) Thruster force vs time

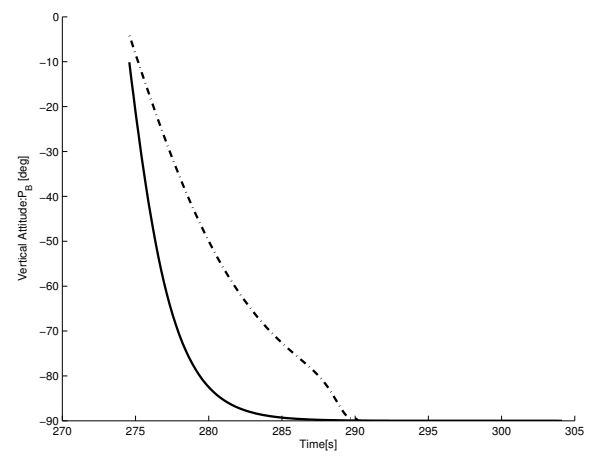

(c) Attitude angle vs time

Fig. 8 Numerical results and Control variables of Gravity turn phase in the case of $30 \%$ thruster failure at the midpoint(-., FGC $(\delta(t)=0 \mathrm{~s}) ;-$, DAC $)$ 
locity is $[0,0,1.67]^{\top} \mathrm{m} / \mathrm{s}$ for FGC with $\delta(t)=0 \mathrm{~s}$, and $[0,0,0.31]^{\top} \mathrm{m} / \mathrm{s}$ for DAC. Therefore DAC is more robust than FGC, as the lander can only survive this failure scenario when controlled using DAC. In this paper, analytical trajectory and embedded autonomy are mainly studied. Compared to other approaches $[10-12,14]$, the DAC can cope with thruster failure and increase the probability of lander survival. In addition, the convergence problem in the tangent guidance law is also overcame since the estimation of time to go $\left(t_{g o}\right)[10,14]$ is not required and the DAC is proved to be convergent using Lyapunov stability theory. Therefore, the approach in this paper introduces and enables the concept of embedded autonomy to mission critical control systems.

\section{CONCLUSIONS}

In this paper, the tangent guidance law, which is derived from optimal control methods, was considered with a closed form solution approached through simplification of the equations of motion by expanding the thrust acceleration and gravitational acceleration, and the cosine of the vertical attitude angle to polynomials. The coefficients of the closed form solution were solved from the boundary conditions. It has been shown that the developed closed form solution is a good match to the numerical solution through comparison with numerical results. It is noted that the closed form nature of the derived solution makes it suitable for on-board implementation within the guidance system. Subsequently, a robust direct adaptive control law was presented which is proved to be convergent using Lyapunov stability theory, thus overcoming the drawback of convergence in the tangent guidance law. Furthermore the direct adaptive control law is shown to be resilient to variations in the available thrust level. This resilience, in combination with on-board implementation of the derived high-accuracy closed form polynomial solution within the guidance system introduces and enables the concept of embedded autonomy to mission critical control systems as traditional on-board monitoring systems are made redundant. 


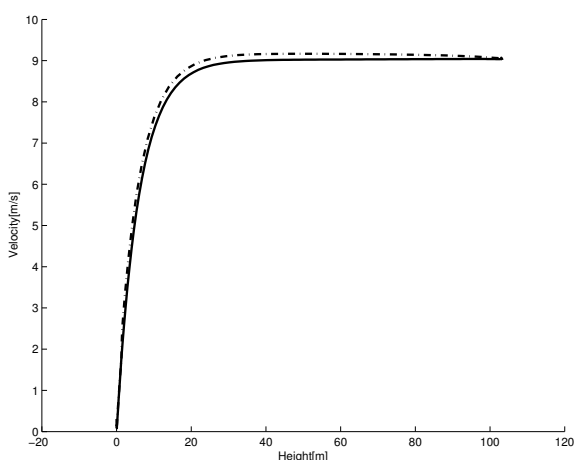

(a) Velocity vs height

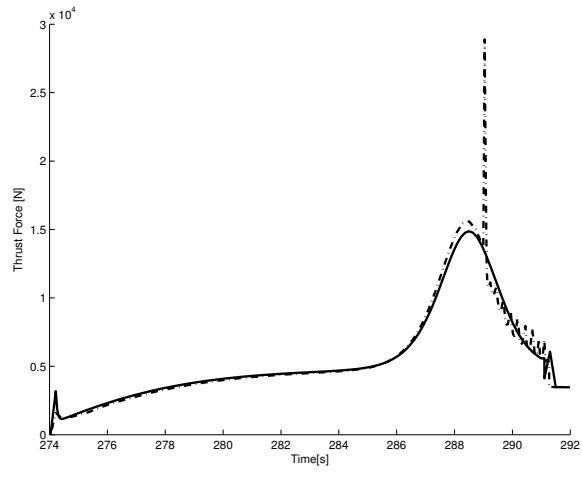

(b) Thruster force vs time

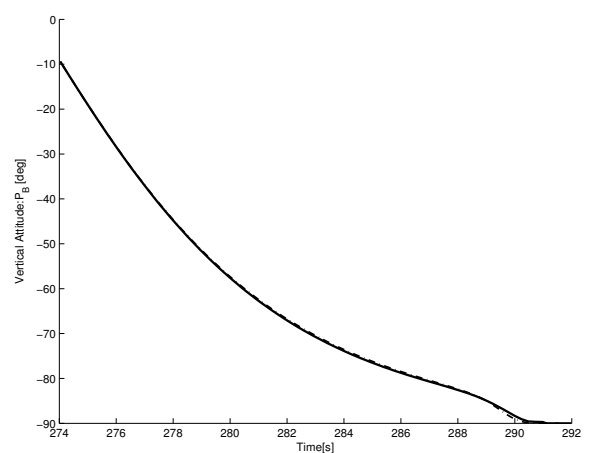

(c) Attitude angle vs time

Fig. 9 Numerical results and control variables for the Gravity turn phase in the case of $30 \%$ thrust failure near the terminal time $(-.$, FGC $(\delta(t)=0 \mathrm{~s}) ;-$, DAC $)$ 
A sample lunar mission scenario was presented in which initial navigation error and thruster failure was considered. Numerical results showed that both fixed gain and direct adaptive control were capable of tracking a required profile if there was enough design margin for the thrusters. Furthermore, direct adaptive control exhibits fast transient response and stability, thus if thruster failure occurs near the terminal time the lander is shown to crash under fixed gain control methods, but survive under direct adaptive control methods. As such, direct adaptive control increases the probability of lander survival while simplifying system level failure, detection, isolation and recovery requirements through the implementation of autonomy in the control loop.

\section{ACKNOWLEDGMENTS}

This work has been supported by the Chinese Nature Science Foundation under grant No. 60535010 and by China Scholarship Council (CSC) Joint-Training Program. Maodeng Li thanks other members of the Advance Space Concepts Laboratory at the University of Strathclyde for help on DAC and numerical simulations. Maodeng Li also thanks Ruikang Li for contributions

\section{REFERENCES}

1 Cheng, R. K. Lunar terminal guidance. Lunar Missions and Exploration, 1964, 1, 308-355.

2 McInnes, C. R. Gravity-turn descent from low circular orbit conditions. $J$. Guid. Control Dyn., 2003, 26(1), 183-185.

3 Chomel, C. T. Development of an analytical guidance algorithm for lunar descent. Ph.D. Dissertation, University of Texas, Austin, TX, 2007.

4 Chomel, C. T. and Bishop, R. H. Analytical lunar descent guidance algorithm. J. Guid. Control Dyn., 2009, 32(3), 915-926. 
5 Citron, S. J. A terminal guidance technique for lunar landing. AIAA J., 1964, 2(3), 503-509.

6 Cheng, R. K and Meredith, C. $\mathbf{M}$ and Conrad, D. A Design considerations for surveyor guidance. J. Spacecraft Rockets, 1966, 3(11), 1569-1576.

7 McInnes, C. R. Non-linear transformation methods for gravity-turn descent. J. Guid. Control Dyn., 1996, 19(1), 247-248.

8 McInnes, C. R. Direct adaptive control for gravity-turn descent. J. Guid. Control Dyn., 1999, 22(2), 373-375.

9 Wang, D. Y. Study guidance and control for lunar soft landing. Ph.D. Dissertation, Harbin Institute of Technology, Harbin, China, 2000.

10 Ueno, S. and Yamaguchi, Y. 3-dimensional near-minimum fuel guidance law of a lunar landing module. In Proceedings of the AIAA Guidance, Navigation and Control Conference, Portland, OR, 1999, pp. 248-257.

11 Afshariand, H. H. and Novinzadeh, A. B. A perturbation approach in determination of closed-loop optimal-fuzzy control policy for planetary landing mission. Proc. IMechE, Part G: J. Aerospace Engineering, 2009, 223(3), 233-243.

12 Sostaric, R. R. and Rea, J. R. Powered descent guidance methods for the moon and mars. In Proceedings of the AIAA Guidance, Navigation and Control Conference, San Francisco, California, 2005.

13 Wang, D. Y. and Huang, X. Y. and Guan, Y. F. GNC system scheme for lunar soft landing spacecraft. Adv. Space Res., 2008, 42(2), 379-385.

14 Sostaric, R. R. Powered descent trajectory guidance and some considerations for human lunar landing. In Proceedings of the AIAA Guidance, Navigation and Control Conference, Breckenridge, Colorado, 2007.

15 Hong, J. and Bhat, S. P. and Bernstein, D. S. Adaptive nonlinear motion control, Internal Rept., Aerospace Engineering Dept., Univ. of Michigan, Ann Arbor,MI, 1998. 
16 Hong, J. and Cummings, I. A. and Bernstein, D. S. Experimental application of direct adaptive control laws for stabilization and command following. In Proceedings of Decision and Control Conference, Phoenix, Arizona USA, IEEE, 1999.

17 Hong, J. and Bernstein, D. S. Adaptive stabilization of non-linear sscillators using direct adaptive control. Int. J. Control, 2001, 74(5), 432-444.

18 Astrom, K. J. and Wittenmark, B. Adaptive control: second edition, 2008 (Dover Publications).

19 Ioannou, P. A. and Sun, J. Robust adaptive control, 1995 (Prentice Hall).

20 Henderson, R. and TEC-SYE CDF study report LES-CTS lunar exploration study cargo transportation system, CDF-38(A), ESA, Inc., Blacksburg, VA, 2005.

21 Franklin, G. F. Feedback control of dynamic systems, 1993 (AddisonWesley Longman Publishing Co., Inc. Boston, MA, USA).

22 Tao, G. and Chen, S. and Tang, X. and Joshi, S. M. Adaptive control of systems with actuator failures, 2004 (Springer Verlag).

23 ECSS Secretariat Space engineering: Ground systems and operations Telemetry and telecommand packet utilization. ECSS-E-70-41A, 2003 (ESA publications division).

\section{List of Figures}

1 Geometry and variables of planetary landing problem . . . . . 5

2 Comparing analytical solution with numerical solution.(--, closed form solution; - , numerical solution) . . . . . . . . . . . 18

3 Landing sites of Monte-carlo simulation result.(Diamond, nominal site; Square, DAC result with 30\% thrust failure; X-mark, FGC result $(\delta(t)=0 \mathrm{~s})$ with $30 \%$ of thrust failure $) \ldots . . . .19$ 
4 Numerical results of optimal phase in the case of $30 \%$ thruster failure at the midpoint(--, required profile; -., FGC with normal gains; - DAC) . . . . . . . . . . . . . 21

5 Control variables of optimal phase in the case of $30 \%$ thruster failure at the midpoint (--, required profile; -., FGC with normal gains; - DAC) . . . . . . . . . . . . . . 22

6 Numerical results for FGC in optimal phase with different values of $\delta(t)$ after thruster failed (--, required profile; -, $\delta(t)=0 \mathrm{~s} ;-$., $\delta(t)=1 \mathrm{~s} ; \ldots, \delta(t)=5 \mathrm{~s}) \ldots \ldots \ldots$

7 Control variables for FGC in optimal phase with different values of $\delta(t)$ after thruster failed (..., required profile; -, $\delta(t)=0 \mathrm{~s} ;-$. , $\delta(t)=1 \mathrm{~s} ;--, \delta(t)=5 \mathrm{~s}) \ldots \ldots \ldots \ldots$

8 Numerical results and Control variables of Gravity turn phase in the case of $30 \%$ thruster failure at the midpoint(-., FGC $\delta(t)=0 \mathrm{~s}) ;-, \mathrm{DAC}) \ldots \ldots \ldots \ldots$

$9 \quad$ Numerical results and control variables for the Gravity turn phase in the case of $30 \%$ thrust failure near the terminal time(-., FGC $(\delta(t)=0 \mathrm{~s}) ;-, \mathrm{DAC})$

\section{APPENDIX}

\section{Notation}




$\begin{array}{ll}\mathbb{F}_{P} & \text { planetary-fixed rotating frame } \\ \mathbb{F}_{H} & \text { lander fixed local vertical frame } \\ g_{0} & \text { gravitational acceleration on the planetary surface } \\ g_{E} & \text { gravitational acceleration on the Earth's surface } \\ h & \text { lander dimensional height above the planetary surface } \\ I_{s p} & \text { lander's specific impulse } \\ m & \text { lander's dimensional mass } \\ m_{0} & \text { lander's initial dimensional mass } \\ r & \text { dimensional distance between planetary center and lander } \\ {[u, v, w]^{\top}} & \text { lander's dimensional velocity } \\ R_{m} & \text { planetary dimensional radius } \\ T & \text { thrust vector magnitude } \\ T_{x}, T_{y}, T_{z} & \text { thrust vector expressed in } \mathbb{F}_{H} \\ \alpha_{B} & \text { lander's vertical attitude angle } \\ \lambda, \phi & \text { planetary longitude and latitude } \\ \mu & \text { planetary gravitational constant } \\ \psi_{B} & \text { lander's horizontal attitude angle } \\ \omega_{P} & \text { planetary angular velocity of rotation } \\ \tilde{\chi} & \end{array}$

